\title{
The lower vitamin C plasma concentrations in elderly men compared with elderly women can partly be attributed to a volumetric dilution effect due to differences in fat-free mass
}

\author{
Alexandra Jungert and Monika Neuhäuser-Berthold* \\ Institute of Nutritional Science, Justus-Liebig-University, Goethestrasse 55, D-35390 Giessen, Germany \\ (Submitted 10 July 2014 - Final revision received 3 January 2015 - Accepted 12 January 2015 - First published online 4 March 2015)
}

\section{Abstract}

Women show higher vitamin $\mathrm{C}$ plasma concentrations than men, but the reasons for this observation still require elucidation. The objective of the present study was to investigate whether sex differences in vitamin C plasma concentrations are present in elderly subjects and whether these differences are due to sex-specific lifestyles, total antioxidant status (TAOS) and/or body composition. Fasting plasma concentrations of vitamin $\mathrm{C}$ were assessed by photometric detection in a cross-sectional study of 181 women and eighty-nine men aged $62-92$ years. Body composition was determined by bioelectrical impedance analysis. Vitamin C intake was assessed with a $3 \mathrm{~d}$ estimated dietary record. Stepwise multiple regression analyses were performed to investigate whether sex is an independent predictor of vitamin $\mathrm{C}$ plasma concentrations by controlling for age, vitamin $\mathrm{C}$ intake, lifestyle factors, TAOS and body composition. Women showed higher vitamin $\mathrm{C}$ plasma concentrations than men $(76 v .62 \mu \mathrm{mol} / 1, P<0.0001)$. In the multiple regression analysis, male sex was a negative predictor of vitamin $C$ plasma concentrations $(\beta=-0 \cdot 214)$, as long as absolute fat-free mass (FFM) was not considered as a confounder. When absolute FFM was included, sex was no longer a predictor of vitamin $C$ plasma concentrations, whereas absolute FFM $(\beta=-0 \cdot 216)$, physical activity level $(\beta=0 \cdot 165)$, intake of vitamin $C$ supplements $(\beta=0 \cdot 164)$, age $(\beta=0 \cdot 147)$ and smoking $(\beta=-0 \cdot 125)$ affected vitamin $C$ plasma concentrations. The results indicate that a higher absolute FFM, and thus a higher distribution volume of vitamin $\mathrm{C}$, contributes to lower vitamin C plasma concentrations in men than women.

Key words: Vitamin C: Sex: Body composition: Fat-free mass

Epidemiological studies have frequently shown higher vitamin C serum/plasma concentrations in women than in men ${ }^{(1-10)}$. These studies generally did not focus on the underlying reasons for the observed sex differences, were mostly conducted in young and middle-aged subjects and were often performed without consideration for confounding variables.

Vitamin C intake and other lifestyle factors, such as smoking behaviour, have been shown to influence vitamin $\mathrm{C}$ serum/ plasma concentrations ${ }^{(4,5,9-12)}$. Additionally, oxidative stress results in an increased consumption of antioxidants, including vitamin $\mathrm{C}^{(13)}$. Therefore, sex differences in those parameters could account for the observed sex differences in vitamin $\mathrm{C}$ status. In addition to those parameters, sex differences in the pharmacokinetics of vitamin $\mathrm{C}$ may exist, seeing that women reach the plasma ascorbate concentration plateau at lower vitamin $\mathrm{C}$ intake levels than men ${ }^{(14)}$. Furthermore, at equal vitamin $\mathrm{C}$ intake levels, women exhibit higher serum/plasma concentrations than men ${ }^{(6,8,9)}$.

In 1991, Blanchard ${ }^{(15)}$ postulated that the sex-related differences in the pharmacokinetics of vitamin $\mathrm{C}$ could be the result of sex differences in body composition. In addition, Sinha et $a l .{ }^{(16)}$ showed that body weight functions as a negative predictor of vitamin C status in middle-aged men. Accordingly, an individual with high amounts of fat-free mass (FFM) can be assumed to have a larger distribution volume of hydrophilic vitamin $\mathrm{C}$ than a person with lower FFM.

The objectives of the present study were (1) to investigate whether sex differences in vitamin $\mathrm{C}$ plasma concentrations are also present in elderly subjects and (2) whether these differences are due to sex-specific lifestyles, total antioxidant status (TAOS) and/or body composition. We concentrated on the hypothesis that sex differences in vitamin $\mathrm{C}$ plasma concentrations might be explained by a higher distribution

Abbreviations: FFM, fat-free mass; GISELA, longitudinal study on nutrition and health status of senior citizens in Giessen; PAL, physical activity level; TAOS, total antioxidant status; TBF, total body fat; WC, waist circumference.

*Corresponding author: M. Neuhäuser-Berthold, fax + 4964199 39069, email monika.neuhaeuser-berthold@ernaehrung.uni-giessen.de 
volume in men than in women, given that men show a higher FFM than women.

\section{Subjects and methods}

\section{Study population}

The subjects were participants in an ongoing cohort study in which the nutrition and health status of independently living senior citizens in Giessen, Germany, has been observed since 1994 at annual intervals and since 1998 at biannual intervals (GISELA study). The main objectives of the GISELA study are to investigate age-related changes in body composition, anthropometric parameters, energy expenditure, bone status, biochemical blood parameters including vitamins, and dietary intake by considering potential confounding variables. The investigations took place at the Institute of Nutritional Science in Giessen, Germany. Subjects were recruited through physicians, advertisements in local newspapers, notices, senior citizen meetings, and word of mouth from subjects who were already participating. Recruitment was continued until 2004, and a total of 584 participants were enrolled. Not all subjects participated in each follow-up. For enrolment, subjects had to be at least 60 years of age and physically mobile. The present study was conducted according to the guidelines laid down in the Declaration of Helsinki, and all procedures involving human subjects were approved by the Ethical Committee of the Faculty of Medicine at the Justus-Liebig-University in Giessen. All subjects provided written informed consent. The present investigation was based on cross-sectional data collected in the follow-up in 2004, in which 350 subjects participated. Subjects with missing data on blood samples, body composition, food record, vitamin C supplement intake and lifestyle factors, including physical activity, alcohol consumption and smoking history, were excluded ( $n$ 79). Additionally, one subject was identified as an outlier regarding vitamin $C$ status (178 $\mu \mathrm{mol} / \mathrm{l})$ and was therefore excluded. Hence, the following analysis included 181 women and eighty-nine men.

\section{Anthropometric data and body composition}

BMI was calculated as the measured body mass (in $\mathrm{kg}$ ) divided by the square of the measured body height (in $\mathrm{m})^{(17)}$. Subjects were classified according to their BMI into normal weight $\left(<25 \cdot 0 \mathrm{~kg} / \mathrm{m}^{2}\right)$, overweight $\left(25 \cdot 0-29 \cdot 9 \mathrm{~kg} / \mathrm{m}^{2}\right)$ or obese $\left(\geq 30 \cdot 0 \mathrm{~kg} / \mathrm{m}^{2}\right)$. Waist circumference (WC) was determined in an upright position by a tape measure to the nearest $1.0 \mathrm{~cm}$. Total body fat (TBF) mass and FFM were recorded with bioelectrical impedance analysis (Akern-RJL BIA 101/S; Data Input) according to the manufacturer's instructions and the predictive formula of Roubenoff et $a l .{ }^{(18)}$. The equation from Roubenoff et al. ${ }^{(18)}$ was chosen because it was derived from a reference population that was comparable in age to the GISELA subjects using similar measurement conditions and because the equation has been validated against the dual-energy X-ray absorptiometry body composition measurements.

\section{Laboratory measurements}

Blood samples were collected after an overnight fast. Following immediate centrifugation, aliquots of heparin plasma, which were acidified with trichloroacetic acid for the determination of vitamin $\mathrm{C}$, and aliquots of EDTA plasma for the determination of TAOS were stored at $-70^{\circ} \mathrm{C}$ until they were analysed after completion of the follow-up. Plasma TAOS, which is inversely related to oxidative stress, was assessed by photometer (Shimadzu UV-160A) ${ }^{(19)}$. Plasma concentrations of vitamin $\mathrm{C}$ were measured by photometer (Beckman Model 35 UV/VIS; Beckman Coulter, Inc.) with a between-days $\mathrm{CV}$ of $3.7 \%^{(20)}$. Vitamin C deficiency was defined as plasma concentrations of $<11.4 \mu \mathrm{mol} / \mathrm{l}$, and plasma concentrations of $<28 \mu \mathrm{mol} / 1$ indicated a moderate risk of developing a deficiency ${ }^{(9)}$. Plasma concentrations of $\geq 50 \mu \mathrm{mol} / 1$ indicated an adequate status ${ }^{(14)}$.

\section{Lifestyle factors}

A validated 3 d estimated dietary record including 146 food items was used to assess the dietary intake of vitamin $\mathrm{C}$ and alcohol $^{(21)}$. The dietary record was handed out to the participants at the date of blood sampling with a request to record their entire food consumption on three consecutive days, starting on a Sunday, directly after each food intake and a request to return the dietary record at their earliest convenience. The majority of dietary records were returned within 3 months. Using self-administered questionnaires, data on age, use of vitamin C supplements, smoking behaviour and physical activity patterns were collected. Subjects who reported using vitamin C supplements sometimes or regularly were classified as 'supplement users'. Smoking behaviour was classified as a dichotomous variable: constant non-smokers $v$. current and ex-smokers. The physical activity level (PAL) of each participant was assessed as described earlier ${ }^{(22)}$.

\section{Statistical analyses}

Statistical analyses were performed using SPSS ${ }^{\circledR}$ version 22.0 for Windows $\left(\mathrm{IBM}^{\circledR}\right)$. Because of non-normally distributed data, the characteristics of the study population are expressed as medians and 25 th -75 th percentiles. Descriptive characteristics were compared between groups via the Mann-Whitney $U$ test or Kruskal-Wallis $H$ test for continuous variables and via the $\chi^{2}$ test for categorical variables.

Factors that potentially influenced vitamin C plasma concentrations were identified by the Spearman correlations. Subsequently, those factors that exhibited a significant association with vitamin $\mathrm{C}$ plasma concentrations were included in the backward stepwise multiple linear regression analysis to ascertain the independent predictors of vitamin $C$ plasma concentrations. A $P$ value of $<0 \cdot 100$ was set as the significance criterion for covariates to remain in the regression model. Considering the observed collinearity of body mass, BMI, WC, TBF and FFM (data not shown), we used separate models. Adjustments for multiple testing were not performed 
due to the exploratory character of the present study. The significance level was set at $P<0 \cdot 05$, and all tests were two-tailed.

\section{Results \\ Characteristics of the study subjects}

The characteristics of the study subjects are shown in Table 1. All except two subjects had vitamin $\mathrm{C}$ plasma concentrations of $>28 \mu \mathrm{mol} / 1$, but $8 \%$ of the women $(n 15)$ and $18 \%$ of the men ( $n$ 16) showed plasma concentrations of $<50 \mu \mathrm{mol} / \mathrm{l}$. Women had significantly higher vitamin $\mathrm{C}$ plasma concentrations and a lower prevalence of a vitamin $\mathrm{C}$ status of $<50 \mu \mathrm{mol} / 1(P=0.019)$ than men. Median vitamin $\mathrm{C}$ intake was slightly under the recommendation of $100 \mathrm{mg} / \mathrm{d}^{(23)}$ in both women and men, and $56 \%$ of the women $(n$ 101) and $64 \%$ of the men $(n 57)$ had an intake of less than $100 \mathrm{mg} / \mathrm{d}$ $(P=0 \cdot 196)$.

Overweight and obesity were noted in 47 and $22 \%$ of the women and in 51 and $17 \%$ of the men, respectively. Vitamin C plasma concentrations, but not vitamin C intake levels, tended to be higher in normal-weight and overweight subjects compared with obese subjects (median $74 v .71 v .62 \mu \mathrm{mol} / \mathrm{l}$; $P=0.088)$. When using tertiles of absolute TBF $(\leq 23.74 v$. $23.75-31.82 v . \geq 31.83 \mathrm{~kg}$ ), neither vitamin $\mathrm{C}$ intake nor vitamin $\mathrm{C}$ plasma concentrations differed between tertiles (data not shown). In contrast, differences in vitamin $\mathrm{C}$ plasma concentrations were observed across tertiles of absolute FFM ( $\leq 39 \cdot 41 v \cdot 39 \cdot 42-49 \cdot 28 v . \geq 49 \cdot 29 \mathrm{~kg}$ ), and vitamin C plasma concentrations were significantly lower in subjects in the highest FFM tertile compared with subjects in the middle or lowest FFM tertile (median $62 v .73 v .76 \mu \mathrm{mol} / 1 ; P<0.001$ ).
Past and current smokers had lower vitamin $\mathrm{C}$ intake (median 86 v. $93 \mathrm{mg} / \mathrm{d} ; \quad P=0.049$ ) and lower vitamin $\mathrm{C}$ plasma concentrations (median $66 v .74 \mu \mathrm{mol} / \mathrm{l} ; \quad P=0.002$ ) than never smokers. Furthermore, in past and current smokers (median 73 v. $62 \mu \mathrm{mol} / 1 ; P=0.005$ ) and in never smokers (median 77 v. $62 \mu \mathrm{mol} / \mathrm{l} ; P=0.015$ ), vitamin $\mathrm{C}$ plasma concentrations, but not vitamin $\mathrm{C}$ intake, were higher in women than in men.

\section{Determinants of vitamin C plasma concentrations in elderly subjects}

The results of the Spearman correlation are given in Table 2. Vitamin C plasma concentrations were positively associated with age, PAL and the use of vitamin $\mathrm{C}$ supplements, and they were negatively correlated with male sex, past or current smoking, body mass, WC and absolute FFM. BMI, absolute TBF, relative FFM, alcohol consumption, dietary intake of vitamin $\mathrm{C}$ and TAOS exhibited no association with vitamin $\mathrm{C}$ plasma concentrations and therefore were not considered in the following multiple regression analyses.

In the multiple regression analysis, male sex $(\beta=-0 \cdot 214$; $P=0.001)$ was a negative predictor of vitamin $\mathrm{C}$ plasma concentrations, whereas age $(\beta=0.181 ; P=0.002)$, the use of vitamin $C$ supplements $(\beta=0.153 ; P=0.008)$ and PAL $(\beta=0.157 ; P=0.007)$ had a positive impact on vitamin $\mathrm{C}$ plasma concentrations, provided that FFM was not considered in the analysis. Additionally, smoking was entered in the regression model but did not reach statistical significance $(\beta=-0.115 ; P=0.064)$. When absolute FFM was included, FFM and past or current smoking were negative determinants, and age, vitamin $\mathrm{C}$ supplement use and PAL were positive

Table 1. Characteristics of the study population

(Median values, 25th (P25) and 75th (P75) percentiles for continuous variables; absolute and relative frequencies for categorical variables)

\begin{tabular}{|c|c|c|c|c|c|}
\hline & \multicolumn{2}{|c|}{ Women ( $n$ 181) } & \multicolumn{2}{|c|}{ Men ( $n$ 89) } & \multirow[b]{2}{*}{$P^{\star}$} \\
\hline & Median & P25-P75 & Median & P25-P75 & \\
\hline Age (years) & $72 \cdot 0$ & $69 \cdot 0-77 \cdot 0$ & $73 \cdot 0$ & $70 \cdot 0-76 \cdot 5$ & 0.247 \\
\hline Body mass (kg) & $69 \cdot 0$ & $62 \cdot 0-78 \cdot 0$ & $78 \cdot 0$ & $71 \cdot 5-86 \cdot 0$ & $<0.001$ \\
\hline BMI $\left(\mathrm{kg} / \mathrm{m}^{2}\right)$ & $26 \cdot 9$ & $24 \cdot 4-29 \cdot 5$ & $26 \cdot 1$ & $24 \cdot 4-28 \cdot 7$ & 0.251 \\
\hline Waist circumference $(\mathrm{cm})$ & $90 \cdot 0$ & $83 \cdot 0-97 \cdot 0$ & $98 \cdot 0$ & $93 \cdot 0-106 \cdot 5$ & $<0.001$ \\
\hline Total body fat (kg) & $29 \cdot 9$ & $24 \cdot 5-36 \cdot 7$ & $22 \cdot 6$ & $19 \cdot 2-29 \cdot 4$ & $<0.001$ \\
\hline Fat-free mass (kg) & 39.4 & $37 \cdot 3-42 \cdot 1$ & $54 \cdot 8$ & $51 \cdot 7-57 \cdot 7$ & $<0.001$ \\
\hline Fat-free mass (\%) & $57 \cdot 0$ & $53 \cdot 3-61 \cdot 3$ & $70 \cdot 1$ & $66 \cdot 0-73 \cdot 2$ & $<0.001$ \\
\hline Total antioxidant status $(\mathrm{mmol} / \mathrm{l})$ & 1.5 & $1.4-1.6$ & 1.6 & $1.5-1.6$ & 0.002 \\
\hline Plasma vitamin $C(\mu \mathrm{mol} / \mathrm{l}) \dagger$ & $76 \cdot 1$ & $61 \cdot 6-89 \cdot 4$ & $61 \cdot 9$ & $54 \cdot 5-74 \cdot 4$ & $<0.001$ \\
\hline Dietary vitamin C intake $(\mathrm{mg} / \mathrm{d})$ & $92 \cdot 7$ & $69.6-131.9$ & $83 \cdot 8$ & $60 \cdot 9-115 \cdot 8$ & 0.058 \\
\hline Vitamin C supplement usersł & \multirow{2}{*}{\multicolumn{2}{|c|}{21}} & & & 0.929 \\
\hline$n$ & & & \multicolumn{2}{|r|}{10} & \\
\hline$\%$ & \multicolumn{2}{|c|}{11.6} & \multicolumn{2}{|c|}{$11 \cdot 2$} & \\
\hline Alcohol intake (g/d) & $1 \cdot 6$ & $0.1-6.7$ & $8 \cdot 0$ & $0 \cdot 4-17 \cdot 2$ & $<0.001$ \\
\hline Physical activity level & $1 \cdot 7$ & $1.6-1.8$ & 1.6 & $1.5-1.8$ & 0.116 \\
\hline Current or ex-smokers & & & & & $<0.001$ \\
\hline$n$ & \multicolumn{2}{|c|}{48} & \multicolumn{2}{|r|}{60} & \\
\hline$\%$ & \multicolumn{2}{|c|}{$26 \cdot 5$} & \multicolumn{2}{|c|}{67.4} & \\
\hline
\end{tabular}

* Mann-Whitney $U$ test for continuous variables, $\chi^{2}$ test for categorical variables to investigate differences between women and men.

† To convert $\mu \mathrm{mol} / \mathrm{l}$ to $\mathrm{mg} / \mathrm{dl}$, multiply $\mu \mathrm{mol} / \mathrm{l}$ by 0.0176 .

¥ Median dosage of vitamin C supplements was $120 \mathrm{mg} / \mathrm{d}$ in women and $55 \mathrm{mg} / \mathrm{d}$ in men, respectively, based on vitamin C supplement users only. 
Table 2. Spearman correlations to find variables associated with vitamin C plasma concentrations

\begin{tabular}{lcc}
\hline Variables & $r_{\mathrm{S}}$ & $P$ \\
\hline Sex (male) & -0.276 & $<0.0001$ \\
Age (years) & 0.143 & 0.018 \\
Body mass $(\mathrm{kg})$ & -0.248 & $<0.0001$ \\
BMI (kg/m $)$ & -0.104 & 0.087 \\
Waist circumference $(\mathrm{cm})$ & -0.270 & $<0.001$ \\
Total body fat $(\mathrm{kg})$ & -0.033 & 0.594 \\
Fat-free mass $(\mathrm{kg})$ & -0.305 & $<0.0001$ \\
Fat-free mass $(\%)$ & -0.106 & 0.083 \\
Total antioxidant status $(\mathrm{mmol} / \mathrm{l})$ & -0.033 & 0.588 \\
Dietary vitamin C intake $(\mathrm{mg} / \mathrm{d})$ & 0.060 & 0.329 \\
Vitamin C supplement use & 0.146 & 0.016 \\
Alcohol intake (g/d) & -0.049 & 0.426 \\
Physical activity level & 0.163 & 0.007 \\
Past or current smoking & -0.187 & 0.002 \\
\hline
\end{tabular}

* Dummy variable (no/yes).

determinants of plasma vitamin $\mathrm{C}$ concentrations, whereas sex was no longer an independent predictor of vitamin $\mathrm{C}$ plasma concentrations (Table 3). When FFM was replaced by body mass, BMI, WC or TBF, male sex remained negatively associated with vitamin $\mathrm{C}$ plasma concentrations (data not shown). Each regression model explained approximately $14 \%$ of the variance in vitamin $\mathrm{C}$ plasma concentrations. We repeated these analyses by excluding subjects who used anti-diabetics ( $n$ 15) or thyroid hormones ( $n$ 81), because they differed in their vitamin $\mathrm{C}$ plasma concentrations from subjects who did not use such drugs (data not shown). As a result, absolute FFM $(\beta=-0.346 ; \quad P<0.0001)$, age $(\beta=0.187 ; \quad P=0.007)$, use of vitamin $C$ supplements $(\beta=0.220 ; P=0.001)$ and PAL $(\beta=0 \cdot 127 ; P=0 \cdot 064)$, but not sex, were predictors of vitamin C plasma concentrations $\left(R^{2} 0 \cdot 189\right)$. Smoking and PAL were no longer significant independent predictors, possibly because the sample size was markedly reduced ( $n$ 178). When FFM was replaced by body mass, BMI, WC or TBF, male sex remained a negative predictor of vitamin $\mathrm{C}$ plasma concentrations (data not shown).

\section{Discussion}

The results of the present study indicate that lower vitamin C plasma concentrations in men compared with women are predominantly explained by higher FFM in men. A special feature of the present approach was the consideration of a variety of potential confounders, such as age, anthropometric variables, body composition, TAOS, vitamin $\mathrm{C}$ intake, alcohol consumption, smoking and PAL.

The present study demonstrates that male sex was only a predictor of vitamin $\mathrm{C}$ plasma concentrations when FFM was not considered. The inclusion of TBF or anthropometric parameters in the regression model did not influence the association between sex and vitamin $\mathrm{C}$ plasma concentrations.

Previous studies considered only anthropometric parameters or TBF, but not FFM, as potential determinants of vitamin $\mathrm{C}$ status. In several studies that were conducted in young and middle-aged subjects, a negative association between BMI and vitamin C serum/plasma concentrations was reported ${ }^{(4,24-26)}$. Furthermore, the relative body fat content ${ }^{(25,26)}$ and anthropometric indices of fat distribution ${ }^{(24-26)}$, such as WC and waist:ratio, were associated with vitamin C status in some, but not all, studies. However, these findings were often controlled for limited confounders ${ }^{(9,25,26)}$ or vanished after body mass was taken into account ${ }^{(25)}$. A study conducted in 545 Spanish subjects who were $\geq 65$ years of age ${ }^{(6)}$ and a study with 979 Canadian subjects who were 20-29 years of age ${ }^{(8)}$ could not verify an independent association between BMI categories and vitamin C serum/ plasma concentrations. In the present study, TBF, BMI and WC were not predictors of vitamin $C$ plasma concentrations, and vitamin $\mathrm{C}$ plasma concentrations did not differ among BMI groups or tertiles of absolute TBF. However, vitamin $\mathrm{C}$ plasma concentrations varied by tertiles of absolute FFM. These observations support the hypothesis that FFM, and not TBF, determines vitamin $\mathrm{C}$ plasma concentrations.

In addition to FFM playing a role in determining vitamin $\mathrm{C}$ plasma concentrations, we initially assumed that the frequently observed lower vitamin $\mathrm{C}$ status in men as compared to women might be attributed to a lower intake of vitamin $\mathrm{C}$, lower TAOS, higher consumption of alcohol or higher proportion of smokers in male subjects. However, sex remained a predictor of vitamin C plasma concentrations after controlling for these factors. Consequently, these factors seem unlikely to be the major contributing factors for the observed impact of sex on vitamin $\mathrm{C}$ status in independently living elderly subjects. Nonetheless, in addition to absolute FFM, current or past smoking was negatively associated with vitamin $\mathrm{C}$ plasma concentrations, whereas age, PAL and the use of vitamin C supplements were positively associated with vitamin C plasma concentrations in the present study population.

In previous studies, smoking was associated with a lower vitamin $C$ status in middle-aged ${ }^{(3-5,9,12)}$ and elderly $(3,6,9,10)$ subjects, which is in accordance with the present results. The lower status in smokers may be a consequence of greater metabolic turnover of ascorbic acid due to an increased production of free radicals as a result of cigarette smoke rather than lower vitamin $\mathrm{C}$ intake $\mathrm{i}^{(4,11)}$.

In agreement with the present results, in the Third National Health and Nutrition Examination Survey (NHANES III), Hampl et $a l^{(3)}$ found a lower prevalence of vitamin $\mathrm{C}$

Table 3. Multiple regression analysis to identify independent determinants of vitamin C plasma concentrations in the elderly $(n 270)^{*}$

\begin{tabular}{lccc}
\hline \multicolumn{4}{c}{$\beta$ (non- } \\
Determinants & standardised) & $\beta$ (standardised) & $P$ \\
\hline Intercept & 22.481 & & 0.307 \\
Age (years) & 0.522 & 0.147 & 0.011 \\
Physical activity level & 21.328 & 0.165 & 0.004 \\
Smoking Past or current & -5.065 & -0.125 & 0.040 \\
$\quad$ smoking (no/yes) & 10.247 & 0.164 & 0.004 \\
$\quad$ Vitamin C & & & \\
$\quad$ supplements (no/yes) & -0.518 & -0.216 & $<0.001$ \\
Fat-free mass (kg) & &
\end{tabular}

* Multiple linear regression analysis using backward stepwise procedure and vitamin C plasma concentrations in $\mu \mathrm{mol} / \mathrm{l}$ as the dependent variable. Only variables of the final model are presented. Variables that were entered into the stepwise regression analysis but did not remain in the final model included sex (female/male). The adjusted coefficient of determination $\left(R^{2}\right)$ for the final model was 0.143 . 
deficiency in subjects aged $\geq 65$ years than in younger subjects. By contrast, in populations with a high prevalence of vitamin $\mathrm{C}$ deficiency, a negative relationship between age and vitamin $\mathrm{C}$ status has been reported ${ }^{(10)}$, whereas some studies of middle-aged ${ }^{(4,5)}$ and elderly subjects ${ }^{(6)}$ show no association. In the NHANES 2003-4, the mean vitamin C serum concentration declined during adolescence, then reached a plateau and increased with advancing age ${ }^{(9)}$. Therefore, the authors suggested a non-linear relationship ${ }^{(9)}$. However, previous studies frequently did not control for relevant confounders, such as FFM or vitamin $\mathrm{C}$ intake. One reason for our observation may be the age-related decline in FFM $\left(r_{\mathrm{S}}-0.116 ; P=0.056\right)$ and concurrent increase in vitamin $\mathrm{C}$ intake $\left(r_{\mathrm{S}} 0 \cdot 214 ; P<0 \cdot 001\right)$ that was noticed in the GISELA subjects. However, age remained a positive predictor of vitamin C plasma concentrations after controlling for FFM and vitamin $\mathrm{C}$ intake in the present study. In addition to a decline in FFM, old age has also been associated with a decrease in FFM hydration ${ }^{(27)}$. Thus, a decrease in fluid volume might further explain the increase in vitamin $\mathrm{C}$ plasma concentration that occurs with advancing age.

To the best of our knowledge, until now, the impact of PAL on vitamin $\mathrm{C}$ plasma concentrations in elderly subjects has not been investigated. In the present study, regular physical activity was associated with higher vitamin C plasma concentrations, even after applying multiple adjustments, including FFM. The reason for this finding is unclear. One may speculate that subjects who perform regular physical activity might have better health, better nutritional conditions and concomitant antioxidant status. The fact that TAOS showed no direct association with vitamin C plasma concentrations in the present study population could be ascribed to a limitation of the method, i.e. the exclusive measurement of the scavenging of the non-physiologic 2,2'-azino-bis-3-ethylbensthiazoline-6sulfonic acid radical; thus, other reactive oxygen species are not considered by this approach ${ }^{(28)}$.

In contrast to previous investigations ${ }^{(3,4,6,9)}$, dietary vitamin C intake was not associated with vitamin C plasma concentrations in the present cohort. Vitamin $\mathrm{C}$ intake was also only moderately associated with vitamin C plasma concentrations in a meta-analysis of twenty-six epidemiological studies with subjects who were $<65$ years of age ${ }^{(29)}$. Studies that have shown an association between vitamin $\mathrm{C}$ intake and serum/plasma concentrations were often performed with middle-aged subjects ${ }^{(4)}$, subjects with lower vitamin C status $^{(3,6,9)}$ and/or higher vitamin $C$ intake levels ${ }^{(3,6)}$ as compared to the GISELA subjects, or they analysed dichotomous variables $^{(9)}$. The association of vitamin $\mathrm{C}$ intake with vitamin C plasma and tissue concentrations follows a sigmoidal relationship ${ }^{(30)}$, i.e. the association appears to approximate an asymptote at plasma concentrations of $>50 \mu \mathrm{mol} / \mathrm{l}^{(14)}$. In the present study, because the median vitamin $C$ plasma concentrations were 76 and $62 \mu \mathrm{mol} / \mathrm{l}$ in women and men, respectively, the concentrations might have reached a plateau; thus, further increase may rely on a higher intake dosage. This could explain why the use of vitamin $\mathrm{C}$ supplements positively affected vitamin $\mathrm{C}$ plasma concentrations in the present subjects despite the relatively low proportion of supplement users as compared with other studies, in which $37-47 \%$ of the subjects consumed supplements ${ }^{(9)}$.

For the first time, the present study shows that sex differences in vitamin $\mathrm{C}$ plasma concentrations are related to differences in FFM. However, some limitations have to be considered. As with any cross-sectional study, causal inferences cannot be derived. Further limitations include the sample size, the imbalanced sex ratio and the use of self-reported data on dietary intake, smoking behaviour and physical activity. Likewise, dietary vitamin $\mathrm{C}$ intake was recorded after blood sampling was performed, which may have biased the association between vitamin $\mathrm{C}$ intake and vitamin $\mathrm{C}$ plasma concentrations. Furthermore, the number of current smokers was small in the present study ( $n$ 12), which may have attenuated the association between smoking and vitamin C plasma concentrations.

Even though we considered a variety of potentially confounding factors, we cannot exclude the possibility that other confounding factors may have influenced the results. The observation in the present study that only $14 \%$ of the variability in vitamin $C$ plasma concentrations were explained by FFM, age, the use of vitamin $C$ supplements and PAL may be ascribed to the rather homogeneous study population with regard to these variables. It should also be mentioned that the GISELA subjects were volunteers, exhibited a higher level of education, consumed lower quantities of alcohol and were less often smokers than their peers in the general German population, and the generalisability of the present findings should therefore be confirmed in other populations.

Future studies should elucidate whether the relationship between FFM and vitamin C plasma concentrations depends only on distribution volume or possibly also on metabolic aspects related to FFM. Moreover, the question arises of whether the differences in plasma concentrations that are caused by differences in body composition are also observed in other indicators of vitamin $\mathrm{C}$ status, such as vitamin $\mathrm{C}$ concentrations in leucocytes or functional markers.

In conclusion, the present investigation provides an explanation for the frequently noticed sex differences in vitamin $\mathrm{C}$ status. The higher absolute FFM and the consequential higher distribution volume in men contribute to lower vitamin $\mathrm{C}$ plasma concentrations in men as compared with women. Therefore, absolute FFM should be considered in the interpretation of plasma vitamin $\mathrm{C}$ concentrations when vitamin $\mathrm{C}$ status and the impact of vitamin $\mathrm{C}$ on health status are analysed. Whether lower vitamin $\mathrm{C}$ plasma concentrations in men are associated with health consequences warrants further investigation.

\section{Acknowledgements}

The authors thank all participants in the GISELA study as well as the staff of the Department of Human Nutrition who helped in the data acquisition.

The present investigation received no specific grant from any funding agency in the public, commercial or non-profit sectors.

The authors' responsibilities are as follows: A. J. performed the statistical analysis, interpreted the data and wrote the 
manuscript. M. N.-B. formulated the research question, designed the study, conducted the research, proofread the manuscript and had primary responsibility for final content. All authors read and approved the final manuscript.

None of the authors had a conflict of interest.

\section{References}

1. Birlouez-Aragon I, Delcourt C, Tessier F, et al. (2001) Associations of age, smoking habits and diabetes with plasma vitamin C of elderly of the POLA study. Int J Vitam Nutr Res 71, 53-59.

2. Olmedilla B, Granado F, Southon S, et al. (2001) Serum concentrations of carotenoids and vitamins $\mathrm{A}, \mathrm{E}$, and $\mathrm{C}$ in control subjects from five European countries. Br J Nutr 85, $227-238$.

3. Hampl JS, Taylor CA \& Johnston CS (2004) Vitamin C deficiency and depletion in the United States: the Third National Health and Nutrition Examination Survey, 1988 to 1994. Am J Public Health 94, 870-875.

4. Galan P, Viteri FE, Bertrais S, et al. (2005) Serum concentrations of beta-carotene, vitamins $\mathrm{C}$ and $\mathrm{E}$, zinc and selenium are influenced by sex, age, diet, smoking status, alcohol consumption and corpulence in a general French adult population. Eur J Clin Nutr 59, 1181-1190.

5. Faure H, Preziosi P, Roussel AM, et al. (2006) Factors influencing blood concentration of retinol, alpha-tocopherol, vitamin $\mathrm{C}$, and beta-carotene in the French participants of the SU.VI.MAX trial. Eur J Clin Nutr 60, 706-717.

6. Vioque J, Weinbrenner T, Asensio L, et al. (2007) Plasma concentrations of carotenoids and vitamin $\mathrm{C}$ are better correlated with dietary intake in normal weight than overweight and obese elderly subjects. Br J Nutr 97, 977-986.

7. Mosdøl A, Erens B \& Brunner EJ (2008) Estimated prevalence and predictors of vitamin C deficiency within UK's low-income population. J Public Health (Oxf) 30, 456-460.

8. Cahill L, Corey PN \& El-Sohemy A (2009) Vitamin C deficiency in a population of young Canadian adults. $\mathrm{Am} \mathrm{J}$ Epidemiol 170, 464-471.

9. Schleicher RL, Carroll MD, Ford ES, et al. (2009) Serum vitamin $\mathrm{C}$ and the prevalence of vitamin $\mathrm{C}$ deficiency in the United States: 2003-2004 National Health and Nutrition Examination Survey (NHANES). Am J Clin Nutr 90, $1252-1263$.

10. Ravindran RD, Vashist P, Gupta SK, et al. (2011) Prevalence and risk factors for vitamin $\mathrm{C}$ deficiency in north and south India: a two centre population based study in people aged 60 years and over. PLOS ONE 6, e28588.

11. Alberg A (2002) The influence of cigarette smoking on circulating concentrations of antioxidant micronutrients. Toxicology 180, 121-137.

12. Dietrich M, Block G, Norkus EP, et al. (2003) Smoking and exposure to environmental tobacco smoke decrease some plasma antioxidants and increase gamma-tocopherol in vivo after adjustment for dietary antioxidant intakes. Am J Clin Nutr 77, 160-166.

13. Stephens JW, Khanolkar MP \& Bain SC (2009) The biological relevance and measurement of plasma markers of oxidative stress in diabetes and cardiovascular disease. Atherosclerosis 202, 321-329.

14. European Food Safety Authority Panel on Dietetic Products, Nutrition and Allergies (EFSA NDA Panel) (2013) Scientific opinion on dietary reference values for vitamin C. EFSA J 11, 3418.

15. Blanchard J (1991) Effects of gender on vitamin C pharmacokinetics in man. J Am Coll Nutr 10, 453-459.

16. Sinha R, Block G \& Taylor PR (1992) Determinants of plasma ascorbic acid in a healthy male population. Cancer Epidemiol Biomarkers Prev 1, 297-302.

17. Jungert A, Roth HJ \& Neuhäuser-Berthold M (2012) Serum 25-hydroxyvitamin $\mathrm{D}_{3}$ and body composition in an elderly cohort from Germany: a cross-sectional study. Nutr Metab (Lond) 9, 42.

18. Roubenoff R, Baumgartner RN, Harris TB, et al. (1997) Application of bioelectrical impedance analysis to elderly populations. J Gerontol A Biol Sci Med Sci 52, M129-M136.

19. Miller NJ, Rice-Evans C, Davies MJ, et al. (1993) A novel method for measuring antioxidant capacity and its application to monitoring the antioxidant status in premature neonates. Clin Sci (Lond) 84, 407-412.

20. Speitling A, Hüppe R, Kohlmeier M, et al. (1992) Bestimmung von Vitamin C im Blutplasma (Determination of vitamin C plasma concentrations). In Methodenhandbuch der Verbundstudie Ernährungserbebung und Risikofaktoren Analytik, VERA - Schriftenreibe (VERA Study - Manual of the Dietary Assessment and Risk Factor Analytic), pp. 100-102 [W Kübler, HJ Anders, W Heeschen and M Kohlmeier, editors]. Niederkleen: Wissenschaftlicher Fachverlag Dr. Fleck.

21. Lührmann PM, Herbert BM, Gaster C, et al. (1999) Validation of a self-administered 3-day estimated dietary record for use in the elderly. Eur J Nutr 38, 235-240.

22. Krems C, Lührmann PM \& Neuhäuser-Berthold M (2004) Physical activity in young and elderly subjects. J Sports Med Phys Fitness 44, 71-76.

23. Deutsche Gesellschaft für Ernährung, Österreichische Gesellschaft für Ernährung, Schweizerische Gesellschaft für Ernährungsforschung, et al. (2013) Referenzwerte für die Nährstoffzufubr (The Reference Values for Nutrient Intake), 1st ed., pp. 167-174. Neustadt a.d. Weinstrasse: Neuer Umschau Buchverlag.

24. Canoy D, Wareham N, Welch A, et al. (2005) Plasma ascorbic acid concentrations and fat distribution in 19,068 British men and women in the European Prospective Investigation into Cancer and Nutrition Norfolk cohort study. Am J Clin Nutr 82, 1203-1209.

25. Johnston CS, Beezhold BL, Mostow B, et al. (2007) Plasma vitamin $\mathrm{C}$ is inversely related to body mass index and waist circumference but not to plasma adiponectin in nonsmoking adults. J Nutr 137, 1757-1762.

26. García OP, Ronquillo D, Caamaño Mdel C, et al. (2012) Zinc, vitamin $\mathrm{A}$, and vitamin $\mathrm{C}$ status are associated with leptin concentrations and obesity in Mexican women: results from a cross-sectional study. Nutr Metab (Lond) 9, 59.

27. Virgili F, D'Amicis A \& Ferro-Luzzi A (1992) Body composition and body hydration in old age estimated by means of skinfold thickness and deuterium dilution. Ann Hum Biol 19, 57-66.

28. Strube M, Haenen GR, Van Den Berg H, et al. (1997) Pitfalls in a method for assessment of total antioxidant capacity. Free Radic Res 26, 515-521.

29. Dehghan M, Akhtar-Danesh N, McMillan CR, et al. (2007) Is plasma vitamin $\mathrm{C}$ an appropriate biomarker of vitamin $\mathrm{C}$ intake? A systematic review and meta-analysis. Nutr J 6, 41.

30. Padayatty SJ, Katz A, Wang Y, et al. (2003) Vitamin C as an antioxidant: evaluation of its role in disease prevention. J Am Coll Nutr 22, 18-35. 